\title{
Gate-induced interlayer asymmetry in ABA-stacked trilayer graphene
}

\author{
Mikito Koshino ${ }^{1}$ and Edward McCann ${ }^{2}$ \\ ${ }^{1}$ Department of Physics, Tokyo Institute of Technology, \\ 2-12-1 Ookayama, Meguro-ku, Tokyo 152-8551, Japan \\ ${ }^{2}$ Department of Physics, Lancaster University, Lancaster, LA1 4YB, UK
}

\begin{abstract}
We calculate the electronic band structure of ABA-stacked trilayer graphene in the presence of external gates, using a self-consistent Hartree approximation to take account of screening. In the absence of a gate potential, there are separate pairs of linear and parabolic bands at low energy. A gate field perpendicular to the layers breaks mirror reflection symmetry with respect to the central layer and hybridizes the linear and parabolic low-energy bands, leaving a chiral Hamiltonian essentially different from that of monolayer or bilayer graphene. Using the self-consistent Born approximation, we find that the density of states and the minimal conductivity in the presence of disorder generally increase as the gate field increases, in sharp contrast with bilayer graphene.
\end{abstract}

PACS numbers: 71.20.-b,81.05.Uw,73.63.-b,73.43.Cd.

Pioneering experiments [1, 2, 3, 4] demonstrated graphene-based transistors using a back gate to vary the carrier density continuously from electron to hole channels, with a minimal conductivity for nominally-zero carrier density. The switching of a graphene-based transistor would be improved by opening an energy gap between the conduction and valence bands, possibly by lateral confinement of electrons in etched structures [5, 6, , 7, , 8] or by employing gates to induce interlayer asymmetry in bilayer graphene [9, 10, 11, 12, 13, 14, 15, 16]. Recently, experimental attention has turned towards the properties of ABA-stacked trilayer graphene, Fig. [(a), 17, 18, 19]. Theory suggests that the bands are of two separate types 10, 11, 20, 21, 22, 23]: two almost-linear bands reminiscent of the bands in monolayer graphene and four parabolic bands similar to those in bilayer graphene. This raises the expectation that the electronic behavior will display no new features as compared to monolayer or bilayer graphene.

In this paper, we show theoretically that the response of ABA-stacked trilayer graphene to external gate potentials is in fact qualitatively different from that in monoor bi-layer graphene. We use an effective-mass model to self-consistently determine the electronic band structure and we show how the breaking of mirror reflection symmetry by interlayer asymmetry causes hybridization of the linear and parabolic bands. Rather than opening a gap, as in bilayer graphene [9], this leaves two bands near zero energy which support chiral quasiparticles. Employing a self-consistent Born approximation to estimate the minimal conductivity as a function of interlayer asymmetry, we find that the conductivity generally increases as asymmetry increases, in sharp contrast with bilayer graphene as illustrated in Fig. 1(b).

A description of trilayer graphene in the presence of external gates must include two parameters that take into account differences in the potentials $V_{1}, V_{2}$, and $V_{3}$ of the three layers. The first, $\Delta_{1}=-e\left(V_{1}-V_{3}\right) / 2$, describes the average energy difference between each adjacent layer [10, 11, 23], while the second, $\Delta_{2}=-e\left(V_{1}-2 V_{2}+V_{3}\right) / 6$, describes the difference between the energy of the cen-
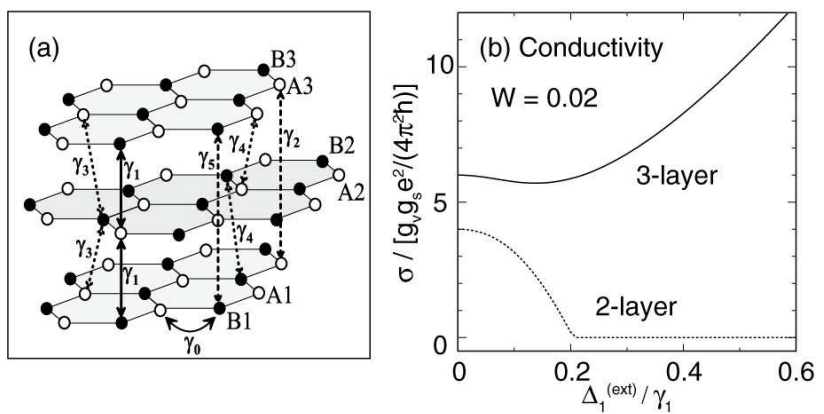

(c)

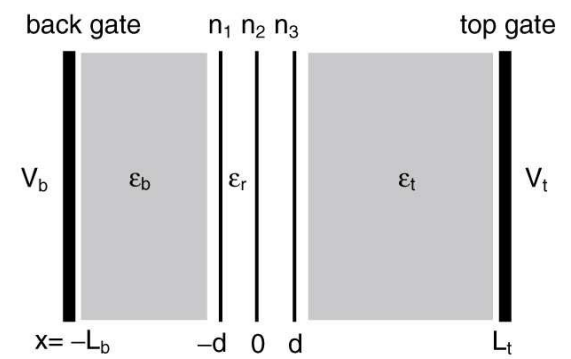

FIG. 1: (a) Schematic of the ABA-stacked trilayer lattice containing six sites in the unit cell, $A$ (white circles) and $B$ (black circles) on each layer, showing the Slonczewski-WeissMcClure parameterization [24] of relevant couplings $\gamma_{0}$ to $\gamma_{5}$. (b) The conductivity versus external asymmetry $\Delta_{1}^{\text {(ext) }}$, calculated for trilayer and bilayer graphene using the self-consistent Born approximation and the band model including $\gamma_{0}$ and $\gamma_{1}$. (c) Schematic of trilayer graphene (three thin black lines at $x=-d, 0, d$ ) with top and bottom gates (thick black lines at $x=L_{t},-L_{b}$ ) separated from the trilayer by dielectric media (gray shaded areas).

tral layer and the average of the outer layers. We model the effect of back and top gates by considering the trilayer as three conducting parallel plates as illustrated in Fig.1(c), with respective electron densities $n_{1}, n_{2}$ and $n_{3}$, located at $x=-d, 0$, and $+d$, respectively, where $d$ is the interlayer spacing, and the permittivity of the trilayer interlayer spaces (without the screening effect of $\pi$-band 
electrons of the trilayer graphene) is $\varepsilon_{r}$. The back (top) gate at $x=-L_{b}\left(x=+L_{t}\right)$, held at potential $V_{b}\left(V_{t}\right)$, is separated from the trilayer by a dielectric medium with relative permittivity $\varepsilon_{b}\left(\varepsilon_{t}\right)$. Using elementary electrostatics, we relate the external gate potentials, the electron densities on the layers, and the interlayer asymmetry parameters:

$$
\begin{aligned}
& \frac{\varepsilon_{b} V_{b}}{L_{b}}+\frac{\varepsilon_{t} V_{t}}{L_{t}}=e\left(n_{1}+n_{2}+n_{3}\right), \\
& \Delta_{1}=\frac{\varepsilon_{t} V_{t}}{L_{t}}-\frac{\varepsilon_{b} V_{b}}{L_{b}}+\frac{e^{2} d}{2 \varepsilon_{r}}\left(n_{1}-n_{3}\right), \\
& \Delta_{2}=-\frac{e^{2} d}{6 \varepsilon_{r}} n_{2} .
\end{aligned}
$$

In the following, we use the total electron density $n_{\text {tot }}=$ $n_{1}+n_{2}+n_{3}$ and $\Delta_{1}^{(\text {ext) }}$ as external parameters instead of $V_{t}$ and $V_{g}$, where $\Delta_{1}^{(\text {ext })}=\varepsilon_{t} V_{t} / L_{t}-\varepsilon_{b} V_{b} / L_{b}$ is the value of $\Delta_{1}$ that would occur if screening were negligible.

We model ABA-stacked trilayer graphene as three coupled honeycomb lattices including pairs of inequivalent sites $\{A 1, B 1\},\{A 2, B 2\}$, and $\{A 3, B 3\}$ in the bottom, center, and top layers, respectively. The layers are arranged according to Bernal $(A-B)$ stacking 24], Fig. 1(a), such that sites $B 1, A 2$, and $B 3$ lie directly above or below each other. We employ an effective-mass model adopting the Slonczewski-Weiss-McClure parameterization, 24] where each parameter is related to relevant coupling in the tight-binding model: $\gamma_{0}$ describes nearestneighbor $(A i-B i$ for $i=\{1,2,3\})$ coupling within each layer, $\gamma_{1}$ describes strong nearest-layer coupling between sites ( $B 1-A 2$ and $A 2-B 3)$ that lie directly above or below each other, $\gamma_{3}\left(\gamma_{4}\right)$ describes weaker nearest-layer coupling between sites $A 1-B 2$ and $B 2-A 3$ ( $A 1-A 2, B 1-B 2$, $A 2-A 3$, and $B 2-B 3)$. With only these couplings, there would be a degeneracy point at each of two inequivalent corners, $K_{ \pm}$, of the hexagonal Brillouin zone [25] but this degeneracy is broken by next-nearest-layer coupling $\gamma_{2}$ (between $A 1$ and $A 3$ ), $\gamma_{5}$ (between $B 1$ and $B 3$ ) and $\delta$, which is the on-site energy difference between $A 1, B 2, A 3$ and $B 1, A 2, B 3$. Note that the parameter $\Delta$ often used in models of three-dimensional (3D) graphite is given by $\Delta=\delta+\gamma_{2}-\gamma_{5}$. In trilayer graphene, the presence of a surface may induce a modification in the value of the band parameters as compared to those in bulk graphite. Here, parameter $\Delta_{2}=-e\left(V_{1}-2 V_{2}+V_{3}\right) / 6$, takes into account a possible difference between the energy of the central layer and the average of the outer layers, and, in general, surface effects may contribute to a non-zero value of $\Delta_{2}$.

In a basis with components $\psi_{A 1}, \psi_{B 1}, \psi_{A 2}, \psi_{B 2}, \psi_{A 3}$, $\psi_{B 3}$, the ABA-stacked trilayer Hamiltonian is

$$
\widetilde{H}=\left(\begin{array}{cccccc}
U_{1} & v \pi^{\dagger} & -v_{4} \pi^{\dagger} & v_{3} \pi & \gamma_{2} / 2 & 0 \\
v \pi & U_{1}+\delta & \gamma_{1} & -v_{4} \pi^{\dagger} & 0 & \gamma_{5} / 2 \\
-v_{4} \pi & \gamma_{1} & U_{2}+\delta & v \pi^{\dagger} & -v_{4} \pi & \gamma_{1} \\
v_{3} \pi^{\dagger} & -v_{4} \pi & v \pi & U_{2} & v_{3} \pi^{\dagger} & -v_{4} \pi \\
\gamma_{2} / 2 & 0 & -v_{4} \pi^{\dagger} & v_{3} \pi & U_{3} & v \pi^{\dagger} \\
0 & \gamma_{5} / 2 & \gamma_{1} & -v_{4} \pi^{\dagger} & v \pi & U_{3}+\delta
\end{array}\right)
$$

where operator $\pi=\xi p_{x}+i p_{y}$ is related to the inplane momentum $\mathbf{p}=\left(p_{x}, p_{y}\right)$ [25], effective velocities are $v=(\sqrt{3} / 2) a \gamma_{0} / \hbar, v_{3}=(\sqrt{3} / 2) a \gamma_{3} / \hbar$, and $v_{4}=$ $(\sqrt{3} / 2) a \gamma_{4} / \hbar, U_{i}=-e V_{i}$, and $\xi= \pm 1$ is the valley index $K_{ \pm}$. Exploiting mirror reflection symmetry of the lattice in the plane of its central layer, Fig. 1(a), we perform a unitary transformation to a basis consisting of linear combinations of the atomic orbitals [22], namely $\left[\psi_{A 1}-\psi_{A 3}\right] / \sqrt{2},\left[\psi_{B 1}-\psi_{B 3}\right] / \sqrt{2},\left[\psi_{A 1}+\psi_{A 3}\right] / \sqrt{2}, \psi_{B 2}$, $\psi_{A 2},\left[\psi_{B 1}+\psi_{B 3}\right] / \sqrt{2}$ :

$$
\begin{aligned}
& H=\left(\begin{array}{cc}
H_{m} & D \\
D^{T} & H_{b}
\end{array}\right), \quad D=\left(\begin{array}{cccc}
\Delta_{1} & 0 & 0 & 0 \\
0 & 0 & 0 & \Delta_{1}
\end{array}\right) \\
& H_{m}=\left(\begin{array}{cc}
\Delta_{2}-\gamma_{2} / 2 & v \pi^{\dagger} \\
v \pi & \Delta_{2}-\gamma_{5} / 2+\delta
\end{array}\right) \text {, } \\
& H_{b}=\left(\begin{array}{cccc}
\Delta_{2}+\gamma_{2} / 2 & \sqrt{2} v_{3} \pi & -\sqrt{2} v_{4} \pi^{\dagger} & v \pi^{\dagger} \\
\sqrt{2} v_{3} \pi^{\dagger} & -2 \Delta_{2} & v \pi & -\sqrt{2} v_{4} \pi \\
-\sqrt{2} v_{4} \pi & v \pi^{\dagger} & -2 \Delta_{2}+\delta & \sqrt{2} \gamma_{1} \\
v \pi & -\sqrt{2} v_{4} \pi^{\dagger} & \sqrt{2} \gamma_{1} & \Delta_{2}+\gamma_{5} / 2+\delta
\end{array}\right)
\end{aligned}
$$

where the average on-site energy $\left[U_{1}+U_{2}+U_{3}\right] / 3$ has been set equal to zero. The Hamiltonian $H$ has a $2 \times 2$ block $H_{m}$ and a $4 \times 4$ block $H_{b}$ on the diagonal, connected by a simple off-diagonal block $D$. Block $H_{m}$ is similar to the Dirac-type Hamiltonian of monolayer graphene and it contributes two bands near zero energy whereas block $H_{b}$ is reminiscent of the Hamiltonian of bilayer graphene [9], except that terms proportional to $\gamma_{1}, \gamma_{3}$, and $\gamma_{4}$ appear with a factor $\sqrt{2}$ [22]. The latter gives two bands split away from zero by energy $\pm \sqrt{2} \gamma_{1}$ and two bands near zero energy.

The monolayer-like block has wave functions possessing odd mirror reflection symmetry, while the wave functions of the bilayer part are even. Since the interlayer asymmetry $\Delta_{1}$ is the only parameter that breaks mirror reflection symmetry, its role is qualitatively different from the other parameters, coupling the monolayer-like and bilayer-like blocks. For large $\Delta_{1}$, two of the lowenergy bands, related to orbitals $\left[\psi_{A 1}-\psi_{A 3}\right] / \sqrt{2}$ and $\left[\psi_{A 1}+\psi_{A 3}\right] / \sqrt{2}$, split away from zero by energy $\epsilon \approx \pm \Delta_{1}$ at the $K$ point, leaving only two bands near zero, associated with $\Psi^{\prime}=\left(\left[\psi_{B 1}-\psi_{B 3}\right] / \sqrt{2}, \psi_{B 2}\right)^{T}$. To obtain an approximate Hamiltonian $H_{\text {eff }}$ for $\Psi^{\prime}$, we denote $H_{2}$ as the diagonal block of Hamiltonian $H$ corresponding to these two low-energy components, $H_{4}$ as the $4 \times 4$ diagonal block corresponding to the high-energy components, and $V$ as the off-diagonal $2 \times 4$ block coupling $H_{2}$ and $H_{4}$. The Schrödinger equation for $\Psi^{\prime}$ can be expanded up to first order in $\varepsilon$ as $\left[H_{2}-V H_{4}^{-1} V^{\dagger}\right] \Psi^{\prime}=\varepsilon S \Psi^{\prime}$ with $S \equiv 1+V H_{4}^{-2} V^{\dagger}$. Then, the effective Hamiltonian for $\Psi=S^{1 / 2} \Psi^{\prime}$ becomes $H_{\text {eff }} \approx S^{-1 / 2}\left[H_{2}-V H_{4}^{-1} V^{\dagger}\right] S^{-1 / 2}$. For the moment, we focus on the role of $\Delta_{1}$ by considering $\Delta_{2}=\gamma_{2}=\gamma_{3}=\gamma_{4}=\gamma_{5}=\delta=0$. For large enough 
$\Delta_{1}\left(\left|\gamma_{1}\right| \gg\left|\Delta_{1}\right| \gg|\epsilon|\right), H_{\text {eff }}$ is written as

$$
\begin{aligned}
H_{\mathrm{eff}} & \approx\left(\begin{array}{cc}
0 & X^{\dagger} \\
X & 0
\end{array}\right) \\
X & =-\frac{\Delta_{1} v \pi}{\sqrt{2} \gamma_{1}}\left(1-\frac{v^{2} \pi \pi^{\dagger}}{\Delta_{1}^{2}}\right)\left(1+\frac{v^{2} \pi \pi^{\dagger}}{\Delta_{1}^{2}}\right)^{-1 / 2} .
\end{aligned}
$$

For plane-wave eigenstates at zero magnetic field, $\pi \pi^{\dagger}$ is just a number, $p^{2}$. The first factor of $\pi=\xi p_{x}+i p_{y}$ in operator $X$ ensures that such eigenstates are chiral, $\Psi=\left(e^{-i \xi \theta / 2}, \mp \xi e^{i \xi \theta / 2}\right)^{T} / \sqrt{2}$ with $\theta=\tan ^{-1}\left(p_{y} / p_{x}\right)$. The expression for the eigenenergies is

$$
\epsilon \approx \pm \frac{v p}{\sqrt{2} \gamma_{1}} \frac{\left(v^{2} p^{2}-\Delta_{1}^{2}\right)}{\sqrt{v^{2} p^{2}+\Delta_{1}^{2}}}
$$

which generalizes Eq. (22) of Ref. 11, showing that there is a small overlap $\delta \epsilon \sim \Delta_{1}^{2} / \gamma_{1}$ between the two lowenergy bands that cross at $p=\Delta_{1} / v$ [11]. This behavior contrasts with that of bilayer graphene, where interlayer asymmetry introduces an energy gap between the lowenergy bands [9] and tends to suppress the chiral nature of quasiparticles in them.

For given parameters $\Delta_{1}, \Delta_{2}$ and fixed total density $n_{\text {tot }}$, the electron densities $n_{1}, n_{2}$ and $n_{3}$ may be determined by summing $\left(\left|\psi_{A i}\right|^{2}+\left|\psi_{B i}\right|^{2}\right) / L^{2}$ over the occupied eigenstates of the Hamiltonian (4). However, such densities are also related to $\Delta_{1}$ and $\Delta_{2}$ through Eqs. (2) and (3), so it is necessary to solve this set of equations selfconsistently in order to obtain values of $\Delta_{1}$ and $\Delta_{2}$ for given external parameters $n_{\text {tot }}$ and $\Delta_{1}^{\text {(ext) }}$. A similar procedure has been applied to bilayer graphene [12, 13, 14] and to many-layered graphene [26]. This Hartree approximation neglects effects including exchange interaction, possible deformation of atomic orbitals in the applied electric field, and the role of $\sigma$ orbitals in screening, but comparison with density functional theory [13] in bilayers suggests that it is qualitatively accurate.

For $\gamma_{2}=\gamma_{3}=\gamma_{4}=\gamma_{5}=\delta=0$ with $n_{\text {tot }}=0$, it is possible to perform a linear response calculation for infinitely small $\Delta_{1}^{\text {(ext) }}$. Within the first order in $\Delta_{1}$, we have $n_{1}-n_{3}=\Pi \Delta_{1}$ with

$$
\Pi=\sum_{\alpha, \alpha^{\prime}} f\left(\varepsilon_{\alpha}\right) \frac{2\left|\left\langle\alpha\left|\frac{\partial H}{\partial \Delta_{1}}\right| \alpha^{\prime}\right\rangle\right|^{2}}{\varepsilon_{\alpha}-\varepsilon_{\alpha^{\prime}}}=-\frac{g_{v} g_{s}}{2 \sqrt{2} \pi} \frac{\gamma_{1}}{(\hbar v)^{2}},
$$

where $g_{s}=g_{v}=2$ are the spin and valley degeneracies, respectively, $|\alpha\rangle$ and $\varepsilon_{\alpha}$ are the eigenstates and eigenenergy of the Hamiltonian without $\Delta_{1}$ or $\Delta_{2}, f(\varepsilon)$ is the Fermi distribution function with zero Fermi energy. Using Eqs. (2) and (3), we obtain the self-consistent solution $\Delta_{1}=\Delta_{1}^{(\text {ext })} / \varepsilon_{\text {eff }}$ with $\varepsilon_{\text {eff }}=1-\left(e^{2} d / 2 \varepsilon_{r}\right) \Pi . \Delta_{2}$ is never induced. Typical parameters $v=1.0 \times 10^{6} \mathrm{~m} / \mathrm{s}, \gamma_{1}=0.4$ $\mathrm{eV}, d=0.334 \mathrm{~nm}, \varepsilon_{r}=2$ give $1 / \varepsilon_{\text {eff }} \approx 0.61$.

To determine the band structure taking into account all the parameters, we find $\Delta_{1}$ and $\Delta_{2}$ self-consistently by employing an iterative numerical approach. We first
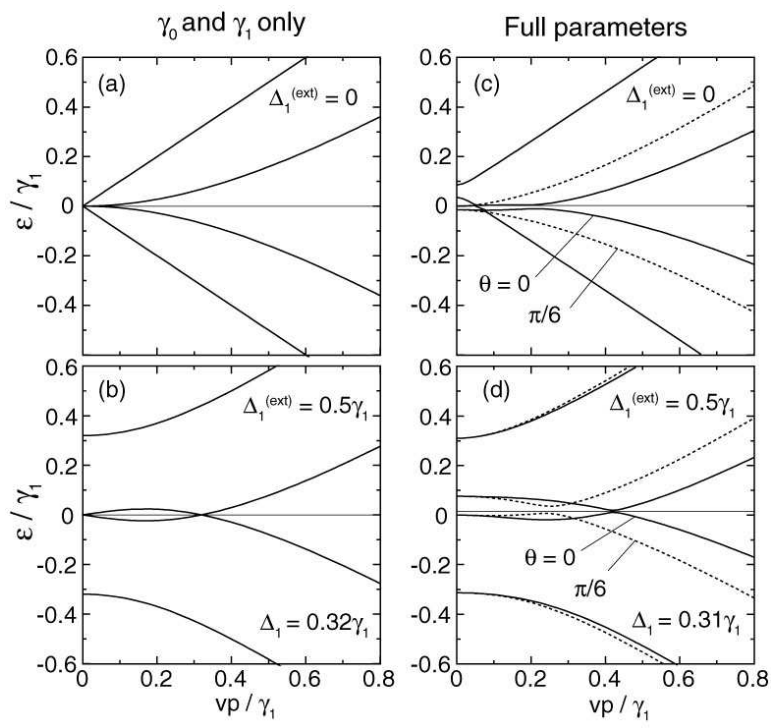

FIG. 2: Self-consistently calculated band structures in trilayer graphene near the $K$ point, with $n_{\text {tot }}=0$. Left plots are for the model including only $\gamma_{0}$ and $\gamma_{1}$, with (a) no asymmetry $\Delta_{1}^{(\text {ext })}=0$ and (b) finite external asymmetry $\Delta_{1}^{(\text {ext })}=0.5 \gamma_{1}$. Right plots are for the full parameter model including $\gamma_{i}(i=1,2, \cdots 5), \delta$, with (c) $\Delta_{1}^{(\text {ext })}=0$ and (d) $0.5 \gamma_{1}$. Dashed and solid curves represent $\theta=0$ and $\pi / 6$. The self-consistently calculated value of $\Delta_{1}$ is shown in the lower side of each plot. The thin horizontal line shows the Fermi energy.

use $\Delta_{1}=\Delta_{1}^{(\text {ext })}$ and $\Delta_{2}=0$ as initial values in the Hamiltonian Eq. (4) and determine the Fermi energy so that the total density is equal to $n_{\text {tot }}$. Then we calculate $n_{i}(i=1,2,3)$ from the occupied eigenstates, which give a new set of $\Delta_{1}$ and $\Delta_{2}$ through Eqs. (2) and (3). We iterate this process until $\Delta_{1}$ and $\Delta_{2}$ converge.

Figure 2 (a) and (c) show the self-consistent band structures at zero external field $\Delta^{\text {(ext) }}=0$ and zero doping $n_{\text {tot }}=0$. To illustrate the role of the extra band parameters we compare (a) the simple model including only $\gamma_{0}, \gamma_{1}$ and (c) the full-parameter model with $\gamma_{2}=-0.05 \gamma_{1}, \gamma_{5}=0.1 \gamma_{1}, \delta=0.125 \gamma_{1}, v_{3}\left(\propto \gamma_{3}\right)=0.1 v$ and $v_{4}\left(\propto \gamma_{4}\right)=0.014 v$ (typical values quoted for bulk graphite [24]). The plots show the vicinity of zero energy, covering the monolayer-like band and the lower branches of the bilayer-like band. In (c), we see that $\gamma_{2}, \gamma_{5}$ and $\delta$ shift the center of the monolayer-like band upward in energy relatively to the bilayer-like band. Also, the trigonal warping effect due to $\gamma_{3}$ is observed as a difference between $\theta=\tan ^{-1}\left(p_{y} / p_{x}\right)=0$ and $\pi / 6$ [9]. Figures [2(b) and 2(d) display the corresponding plots in the presence of a finite external field $\Delta_{1}^{\text {(ext) }}=0.5 \gamma_{1}$. The values of $\Delta_{1}$ determined self-consistently are shown in the lower side of each plot. In every case the screening ratio $\Delta_{1} / \Delta_{1}^{(\text {ext })}$ is about 0.6 , which is close to the linear response theory. For Fig. 2 (b), where only $\gamma_{0}$ and $\gamma_{1}$ are included, there is a small overlap at zero energy described by Eq. (8). In 

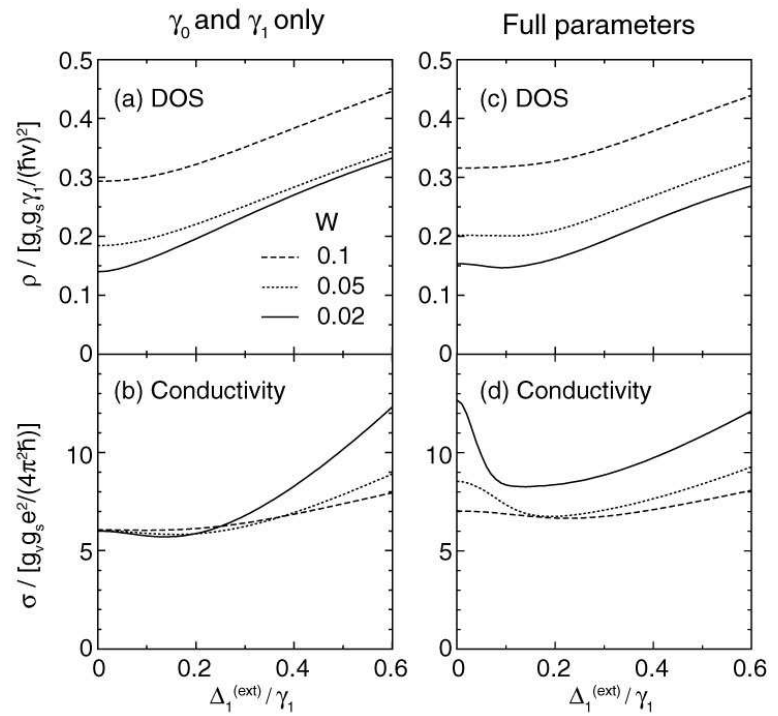

FIG. 3: (a),(c) Density of states and (b),(d) conductivity at $n_{\text {tot }}=0$ as functions of $\Delta_{1}^{(\text {ext })}$ for the model including only $\gamma_{0}$ and $\gamma_{1}$ (left) and the full parameter model (right). Solid, dotted, and dashed lines are for progressively larger disorder strengths.

the full parameter model (d), there is a similar amount of band overlap while the exact magnitude of momentum at the crossing point $v p \sim \Delta_{1}$ varies with angle $\theta$ in a trigonal manner, and there is a tiny gap at those crossing points. In (d), the self-consistent calculations yield tiny $\Delta_{2}<0.01 \gamma_{1}$ due to non-zero $\gamma_{2}, \gamma_{5}$ and $\delta$.

For each band structure we estimate the density of states (DOS) and conductivity using the self-consistent Born approximation [27, 28]. We assume that the scatterers are on-site potentials localized on each layer, which is modeled by $V(\mathbf{r})=\sum_{m=1,2,3} \sum_{i} u_{i} \delta\left(\mathbf{r}-\mathbf{r}_{i}\right) \hat{P}^{(m)}$ where $u_{i}$ and $\mathbf{r}_{i}=\left(x_{i}, y_{i}\right)$ are the amplitude and the twodimensional position of the $i$-th scatterer, respectively, and $\hat{P}^{(m)}$ is the projection operator onto the $m$-th layer. We neglect inter-valley scattering between $K_{ \pm}$. The disorder strength is characterized by $W=n_{\text {imp }} u^{2} /\left(4 \pi \hbar^{2} v^{2}\right)$ [27] where $n_{\mathrm{imp}}$ is the total number of scatterers over all the layers, and $u=\left\langle u_{i}^{2}\right\rangle$. For the model with only $\gamma_{0}$ and $\gamma_{1}$, the energy scale for the level broadening at zero energy is given by $\Gamma \sim(\pi / \sqrt{2}) W \gamma_{1}$. Following Ref. 27, we compute the self-energy and the vertex corrections for the velocity operators, and calculate the conductivity using the Kubo formula.

Figures 3(a) and 3(c) show the DOS as a function of $\Delta_{1}^{\text {(ext) }}$ at $n_{\text {tot }}=0$, for several values of the disorder strength $W$. The left and right panels correspond to the simple model with $\gamma_{0}$ and $\gamma_{1}$, and the full parameter model discussed previously, although the behavior in each case is similar, DOS increases with $\Delta_{1}^{\text {(ext) }}$. Figure 3(b), (d) are plots of the conductivity corresponding to (a),(c) respectively. The general trend is for an increase of conductivity as $\Delta_{1}^{(\text {ext) }}$ increases, ex- cept for the vicinity of $\Delta_{1}^{(\text {ext })}=0$ in panel (d). This may be roughly understood by considering the relation $\sigma=e^{2} \rho_{F} v_{F}^{2} \tau / 2$ with velocity $v_{F}$, DOS $\rho_{F}$ and relaxation time $\tau$ at the Fermi energy. When we assume that all the states on the Fermi energy are equally mixed by disorder, we have $\tau \propto \rho_{F}^{-1}$, suggesting that the conductivity is determined by $v_{F}^{2}$. The dispersion, Eq. (8), approximates, in regions far from the origin $v p \gg\left|\Delta_{1}\right|$, to $\epsilon \approx \pm\left(v^{2} p^{2}-3 \Delta_{1}^{2} / 2\right) /\left(\sqrt{2} \gamma_{1}\right)$, showing that the electron and hole bands are pushed towards zero energy by the introduction of $\Delta_{1}$. This leads to an increase in the expectation value of the band velocity in a disorder-broadened energy window near $\epsilon=0$, and thus the conductivity at the charge neutral point is enhanced.

In the simple $\gamma_{0}-\gamma_{1}$ model, Fig. 3. (b), the conductivity at $\Delta_{1}^{(\text {ext })}=0$ takes a universal value $\sigma=3 g_{v} g_{s} e^{2} /\left(2 \pi^{2} \hbar\right)$ independently of $W$. This is because the Fermi energy coincides with the degeneracy point of the monolayer and bilayer bands, and the value is indeed equal to the summation of the minimum conductivity of monolayer graphene [27] and that of bilayer graphene [28] estimated in the self-consistent Born approximation. In Fig. 3(d), for the full parameter model, the conductivity is largely enhanced around $\Delta_{1}^{(\text {ext })}=0$, because, as observed in Fig. 2(c), the Fermi energy crosses the off-center part of the monolayer-like band making a large contribution to the typical band velocity. The conductivity drops sharply as $\Delta_{1}^{\text {(ext) }}$ grows from zero as the monolayer band is gapped away. When $\Delta_{1}^{(\text {ext })}$ is increased further, the conductivity grows similarly to, but a little more slowly than, Fig. 3(b), because of the tiny gap at the band crossing point observed in Fig. 2(d). The differences between Fig. 3(b) and (d) become smaller for larger $W$, as disorder-broadening masks details dependent on the precise values of band parameters.

To conclude, we have shown that the breaking of mirror reflection symmetry by interlayer asymmetry $\Delta_{1}$ in ABAstacked trilayer graphene causes hybridization of the linear and parabolic bands, leaving just two bands in the vicinity of zero energy. The band hybridization produces an increase in density of states and typical band velocity with asymmetry $\Delta_{1}$, leading to an increase in minimal conductivity in qualitative agreement with recent transport experiments 19]. As demonstrated in Fig. 1(b), which compares the conductivities of trilayer and bilayer graphene, the response of trilayers to gate-induced asymmetry is in sharp contrast with bilayers, where the conductivity is suppressed by a perpendicular electric field owing to the opening of a gap between the electron and hole bands [16].

The authors thank T. Ando, V.I. Fal'ko, and H. Schomerus for discussions, and M.F. Craciun, A.F. Morpurgo, S. Russo, and S. Tarucha for discussions and for sharing their experimental data prior to publication. This project was funded by EPSRC-GB First Grant No. EP/E063519/1, the Royal Society, and the Daiwa Anglo-Japanese Foundation, and by Grants-in-Aid for 
[1] K.S. Novoselov, A.K. Geim, S.V. Morozov, D. Jiang, Y. Zhang, S.V. Dubonos, I.V. Grigorieva, A.A. Firsov, Science 306, 666 (2004).

[2] K.S. Novoselov, A.K. Geim, S.V. Morozov, D. Jiang, M.I. Katsnelson, I.V. Grigorieva, S.V. Dubonos, and A.A. Firsov, Nature 438, 197 (2005).

[3] Y.B. Zhang, Y.W. Tan, H.L. Stormer, P. Kim, Nature 438, 201 (2005).

[4] K. S. Novoselov, E. McCann, S.V. Morozov, V.I. Fal'ko, M.I. Katsnelson, U. Zeitler, D. Jiang, F. Schedin, A.K. Geim, Nat. Phys. 2, 177 (2006).

[5] M.Y. Han, B. Ozyilmaz, Y. Zhang, and P. Kim, Phys. Rev. Lett. 98, 206805 (2007).

[6] F. Miao, S. Wijeratne, Y. Zhang, U. C. Coskun, W. Bao, and C. N. Lau, Science 317, 1530 (2007).

[7] C. Stampfer, J. Guettinger, F. Molitor, D. Graf, T. Ihn, and K. Ensslin, Appl. Phys. Lett. 92, 012102, (2008).

[8] L. A. Ponomarenko, F. Schedin, M. I. Katsnelson, R. Yang, E. H. Hill, K. S. Novoselov, and A. K. Geim, Science 320, 356 (2008).

[9] E. McCann and V.I. Fal'ko, Phys. Rev. Lett. 96, 086805 (2006).

[10] C.L. Lu, C.P. Chang, Y.C. Huang, R.B. Chen, and M.L. Lin, Phys. Rev. B 73, 144427 (2006).

[11] F. Guinea, A.H. Castro Neto, and N.M.R. Peres, Phys. Rev. B 73, 245426 (2006).

[12] E. McCann, Phys. Rev. B 74, 161403(R) (2006).

[13] H. Min, B.R. Sahu, S.K. Banerjee, and A.H. MacDonald, Phys. Rev. B 75, 155115 (2007).

[14] E.V. Castro, K.S. Novoselov, S.V. Morozov, N.M.R. Peres, J.M.B. Lopes dos Santos, J. Nilsson, F. Guinea, A.K. Geim, A.H. Castro Neto, Phys.
Rev. Lett. 99, 216802 (2007).

[15] T. Ohta, A. Bostwick, T. Seyller, K. Horn, and E. Rotenberg, Science 313, 951 (2006).

[16] J.B. Oostinga, H.B. Heersche, X. Liu, A.F. Morpurgo and L.M.K. Vandersypen, Nature Mater. 7, 151 (2008).

[17] T. Ohta, A. Bostwick, J. L. McChesney, T. Seyller, K. Horn, and E. Rotenberg Phys. Rev. Lett. 98, 206802 (2007).

[18] J. Guettinger, C. Stampfer, F. Molitor, D. Graf, T. Ihn, and K. Ensslin, New. J. Phys. 10, 125029 (2008).

[19] M.F. Craciun, S. Russo, M. Yamamoto, J.B. Oostinga, A.F. Morpurgo and S. Tarucha, to be published in Nature Nanotechnology.

[20] S. Latil and L. Henrard, Phys. Rev. Lett. 97, 036803 (2006).

[21] B. Partoens and F.M. Peeters, Phys. Rev. B 74, 075404 (2006); 75, 193402 (2007).

[22] M. Koshino and T. Ando, Phys. Rev. B 76, 085425 (2007); 77, 115313 (2008).

[23] M. Aoki and H. Amawashi, Solid State Commun. 142, 123 (2007).

[24] M.S. Dresselhaus and G. Dresselhaus, Adv. Phys. 51, 1 (2002).

[25] Corners of the hexagonal Brillouin zone are located at wave vector $\mathbf{K}_{\xi}=\xi\left(\frac{4}{3} \pi a^{-1}, 0\right)$, where $\xi= \pm 1$ and $a$ is the lattice constant.

[26] F. Guinea, Phys. Rev. B 75, 235433 (2007).

[27] N.H. Shon and T. Ando, J. Phys. Soc. Jpn. 67, 2421 (1998); Y. Zheng and T. Ando, Phys. Rev. B 65, 245420 (2002).

[28] M. Koshino and T. Ando Phys. Rev. B 73, 245403 (2006). 